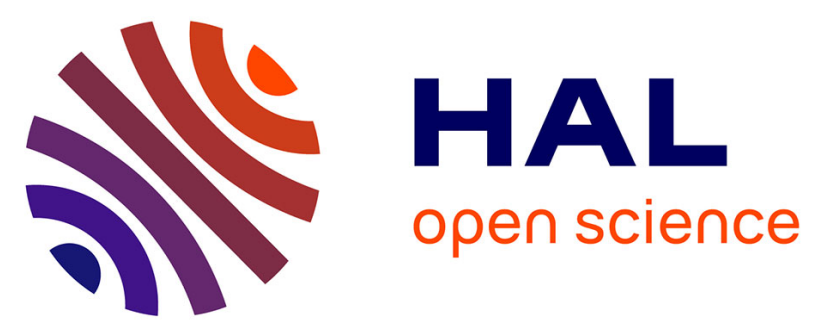

\title{
Characterization and reduction of the amplitude-to-phase conversion effects in telemetry
}

Joffray Guillory, Jorge García-Márquez, Christophe Alexandre, Daniel Truong, Jean-Pierre Wallerand

\section{- To cite this version:}

Joffray Guillory, Jorge García-Márquez, Christophe Alexandre, Daniel Truong, Jean-Pierre Wallerand. Characterization and reduction of the amplitude-to-phase conversion effects in telemetry. Measurement Science and Technology, 2015, 26 (8), pp.084006. 10.1088/0957-0233/26/8/084006 . hal02448935

\section{HAL Id: hal-02448935 \\ https://hal-cnam.archives-ouvertes.fr/hal-02448935}

Submitted on 11 Feb 2020

HAL is a multi-disciplinary open access archive for the deposit and dissemination of scientific research documents, whether they are published or not. The documents may come from teaching and research institutions in France or abroad, or from public or private research centers.
L'archive ouverte pluridisciplinaire HAL, est destinée au dépôt et à la diffusion de documents scientifiques de niveau recherche, publiés ou non, émanant des établissements d'enseignement et de recherche français ou étrangers, des laboratoires publics ou privés. 


\section{Characterization and reduction of the amplitude-to-phase conversion effects in telemetry}

\begin{tabular}{|r|l|}
\hline Journal: & Measurement Science and Technology \\
\hline Manuscript ID: & Draft \\
\hline Danuscript Type: & Special Issue Article \\
\hline Complete List of Authors: & $\begin{array}{l}\text { GUILLORY, Joffray; Cnam, } \\
\text { garcia-marquez, jorge; Cnam, } \\
\text { alexandre, christophe; Cnam, } \\
\text { truong, daniel; Cnam, } \\
\text { Wallerand, Jean-Pierre; CNAM, LCM }\end{array}$ \\
\hline Article Keywords: & $\begin{array}{l}\text { laser diode, absolute distance meter, photodetection, amplitude } \\
\text { modulation, phase measurement, amplitude-to-phase coupling, optical } \\
\text { telemetry }\end{array}$ \\
\hline Abstract: & $\begin{array}{l}\text { We are developing a telemeter based on the measurement of the phase } \\
\text { accumulated by a RF sine wave during its propagation in air. This wave is } \\
\text { carried by a laser beam thanks to an intensity modulation. The main } \\
\text { limitation of this technique lies in amplitude-to-phase conversion occurring } \\
\text { in the detection of this modulation. Therefore, we characterized in this } \\
\text { paper this phenomenon for a given telemetric system and discuss on how } \\
\text { to reduce its effects on the resolution and the accuracy of the distance } \\
\text { measurement. Finally, a solution is implemented and tested outdoor, in } \\
\text { real conditions of use. }\end{array}$ \\
\hline
\end{tabular}




\title{
Characterization and reduction of the amplitude-to-phase conversion effects in telemetry
}

\author{
Joffray Guillory $^{1}$, Jorge García-Márquez ${ }^{1}$, Christophe Alexandre ${ }^{2}$, Daniel Truong ${ }^{1}$ \\ and Jean-Pierre Wallerand ${ }^{1 *}$. \\ ${ }^{1}$ Laboratoire commun de métrologie LNE-CNAM (LCM), LNE, 1 rue Gaston Boissier, 75015 PARIS \\ ${ }^{2}$ Centre d'études et de recherche en informatique et communications (CEDRIC), CNAM, 292 rue Saint-Martin, 75003 PARIS \\ *Corresponding author: jean-pierre.wallerand@cnam.fr
}

Keywords: laser diode, absolute distance meter, photodetection, amplitude modulation, phase measurement, amplitude-to-phase coupling, optical telemetry.

\begin{abstract}
We are developing a telemeter based on the measurement of the phase accumulated by a RF sine wave during its propagation in air. This wave is carried by a laser beam thanks to an intensity modulation. The main limitation of this technique lies in amplitude-to-phase conversion occurring in the detection of this modulation. Therefore, we characterized in this paper this phenomenon for a given telemetric system and discuss on how to reduce its effects on the resolution and the accuracy of the distance measurement. Finally, a solution is implemented and tested outdoor, in real conditions of use.
\end{abstract}

\section{Introduction}

A well-known technique for measuring distances consists in measuring the phase shift accumulated by an intensity-modulated light beam during its propagation in air [1]. This principle is widely implemented, for instance in distance meters for geodetic applications [2] or in absolute trackers for tridimensional measurements in large volumes [3]. We are developing such a telemeter by using common and affordable optoelectronic devices from the telecommunication industry, such as a laser diode at a wavelength of $1550 \mathrm{~nm}$ and an EAM (Electro-Absorption Modulator) that enables high modulation frequency, up to $10 \mathrm{GHz}$ in a simple manner. Thus, the light is intensity modulated by a high RF (Radio Frequency) carrier, propagated in air, and then converted by a photodetector into an electrical signal. The phase accumulated by the sine wave during its propagation is measured from this electrical signal after a frequency down-conversion. This down-conversion can be implemented either in the electrical domain using a classical RF mixer, or in the optical domain [4] using a superheterodyne detection [5-7] or a similar MSTAR (Modulation Sideband Technology for Absolute Ranging) technique [8]. In the latter, extra AOMs (Acousto-Optic Modulator) are used to realize the optical demodulation, which implies a more complex and expensive system. Consequently, we choose the option of a direct photodetection at high frequency with a down-conversion based on a RF mixer. This choice allows us to use off-the-shelf components. Nevertheless, this detection at high frequency exhibits important AM/PM (Amplitude Modulation to Phase Modulation) conversions as soon as the optical power varies [9]. In other words, the photodetection becomes a critical part of the system that worsens the phase noise due to AM/PM coupling.

In this paper, we quantified these AM/PM conversions for three different photodiode technologies in order to identify the optimal conditions for noise rejection in distance measurements. This is essential when the power variations of the detected light beam are 
MacroScale

Recent developments in traceable dimensional measurements Measurement Science and Technology

large, for instance outdoor or in uncontrolled industrial environments. To this end, we studied the dependence of the phase of the RF signal with two prominent parameters: the optical power received by the photodiodes and the bias voltage applied to it. Finally we presented distance measurements realized outdoor with one of the characterized photodiodes. By selecting the amplitudes of the detected telemetric signal, we demonstrated a resolution of $15 \mu \mathrm{m}$ for a distance of $110 \mathrm{~m}$.

\section{Description of the telemeter}

The experimental setup of the developed telemeter is depicted in Fig. 1. A temperaturestabilized DFB (Distributed FeedBack) laser diode at $1550 \mathrm{~nm}$ with a built-in EAM generates an optical signal intensity-modulated at $1310 \mathrm{MHz}$. This signal is boosted by an EDFA (Erbium-Doped Fibre Amplifier) and passes through an optical switch based on MEMS (MicroElectroMechanical Systems) technology. Thanks to this switch, the measurement path is periodically bypassed to use an optical reference path of fixed distance that aims to remove the phase drifts coming from the electronics. On the measurement path, an optical splitter, used as a circulator, guides the light up to a fiber termination. Then a $2.5 \mathrm{~cm}$ diameter offaxis parabolic mirror of $5 \mathrm{~cm}$ focal length collimates the free-space beam for long distance propagation up to a reflective target, which is a hollow corner cube. The reflected beam is then re-injected in the fiber, passes again through the splitter (i.e. the circulator), and is detected by a photodetector. The latter is alternately illuminated by the measurement path or [D1] by the reference path, depending on the optical switch status.

The phase shift is finally measured between the electrical signals coming from the photodetector ( $\left.\phi_{\text {optical measure et }} \phi_{\text {optical reference }}\right)$ and the one modulating the laser diode and

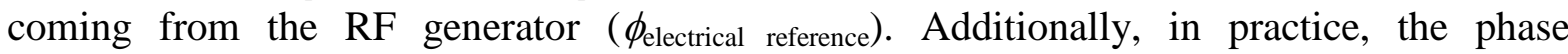
measurement is realized in two steps, between a first corner cube placed close to the telemeter and a second one placed far away, at the target location. Thus, the phase shift $\phi$ is equal to:

$\phi=\left(\phi_{\text {optical }}-\phi_{\text {electrical reference }}\right)_{\text {position2 }}-\left(\phi_{\text {optical }}-\phi_{\text {electrical reference }}\right)_{\text {position1 }}$ with $\phi_{\text {optical }}=\phi_{\text {optical measure }}-\phi_{\text {optical reference }}$ (1)

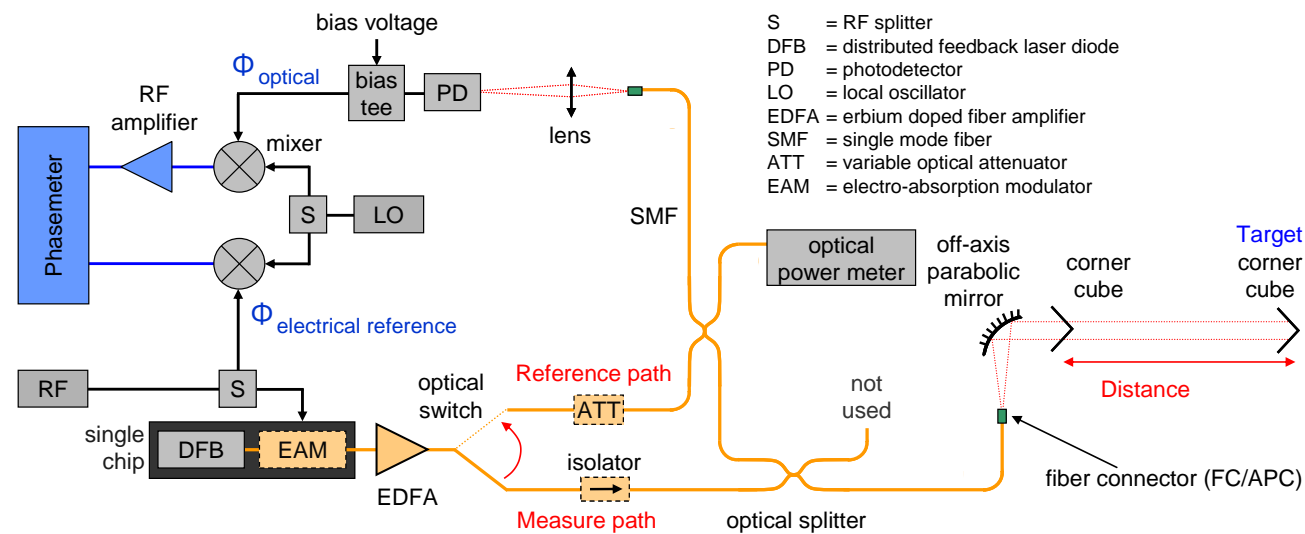

Figure 1: Functional setup of the developed telemeter. Orange lines: fiber-optic light guide; red lines: freespace light; black lines: high frequency electronic; blue lines: intermediate frequency electronic (10 MHz).

Then the phase difference is converted into a distance $D$ as follows:

$$
2 \times D=\left(\frac{\phi}{2 \pi}+k\right) \times \Lambda \text { with } \Lambda=\frac{c}{n \cdot f_{R F}}
$$


where $c$ is the speed of light in vacuum, $n$ the group refractive index of air (fixed to unity in this paper), $f_{\mathrm{RF}}$ the high frequency modulation, and $k$ an integer number corresponding to the number of synthetic wavelengths $\Lambda$ within the distance to be measured. The latter could be determined by a set of measurement at different RF carriers.

The distance resolution is a function of the chosen synthetic wavelength: the higher the modulation frequency, the better the resolution. However, since high frequency signals cannot be directly measured by the phasemeter - based on a FPGA (Field-Programmable Gate Array) and $245 \mathrm{MSa} / \mathrm{s}$ ADCs (Analog to Digital Converters) - a down-conversion at an IF (Intermediate Frequency) of $10 \mathrm{MHz}$ has been implemented before the phase shift measurement. We take advantage of this down-conversion to perform RF amplification at IF and so to be less sensitive to the AM/PM conversions occurring in the electronic stages.

In the phasemeter, a capture of the signals, their digital down-conversions and the calculation of their phase difference are realized every $4 \mathrm{~ms}$. The results are then averaged under Matlab ${ }^{\circledR}$ by block of 50 samples $(50 \times 4 \mathrm{~ms})$ to provide the phase shift measurement. In this configuration and with a RF carrier at $1310 \mathrm{MHz}$, the whole telemeter provides a resolution standard deviation over 100 successive phase shift measurements - of $4 \mu \mathrm{m}(\sim 1 / 60000$ of the synthetic wavelength).

The phasemeter provides also the amplitude of all the signals used for the phase shift measurements. Thus, from the first measurements, we observed a strong correlation between the amplitude variations (recorded at $10 \mathrm{MHz}$ ) of the photodetected signal after propagation in air and the phase shift variations.

\section{Study of the AM/PM coupling}

\section{Characterization of the AM/PM coupling}

We have measured the AM/PM coupling ( $\left.\Delta \Phi_{\mathrm{AM} / \mathrm{PM}}\right)$ occurring in the reception sub-system of our telemeter for three different kinds of free-space InGaAs (Indium Gallium Arsenide) photodetectors - a MSM (Metal-Semiconductor-Metal), an avalanche and a Schottky photodiodes - followed by an electronic stage composed of a mixer, amplifiers and the home-made phasemeter. Table 1 summarizes the characteristics of the tested photodiodes.

\begin{tabular}{llll} 
& \multicolumn{2}{c}{ Table 1: Characteristics of the tested photodiodes. } \\
& InGaAs MSM photodiode & InGaAs avalanche photodiode & InGaAs Schottky photodiode \\
Model & Hamamatsu G7096-03 & Hamamatsu G8931-04 & New Focus 1417 \\
Active Area & $200 \times 200 \mu \mathrm{m}^{2}$ & $40 \mu \mathrm{m}$ diameter & $25 \mu$ m diameter \\
Bias Voltage & up to $15 \mathrm{~V}$ & up to $40 \mathrm{~V}$ & fixed to $9 \mathrm{~V}$ \\
Wavelength range & {$[850-1650] \mathrm{nm}$} & {$[950-1700] \mathrm{nm}$} & {$[950-1650] \mathrm{nm}$} \\
RF Bandwidth & rise / fall times of $40 / 120 \mathrm{ps}$ & $4 \mathrm{GHz}$ & $25 \mathrm{GHz}$ \\
Efficiency & $0.4 \mathrm{~A} / \mathrm{W} @ 1310 \mathrm{~nm}$ & $0.9 \mathrm{~A} / \mathrm{W} @ 1550 \mathrm{~nm}$ & -
\end{tabular}

The characterization of the reception sub-system was realized using the setup depicted in Fig. 2. We have used the same laser diode and the same optical modulator as the ones described above, and we have added a fibered AOM, used as an optical attenuator, to simulate an amplitude noise. In fact, the power driving the AOM at $80 \mathrm{MHz}$ was square-wave modulated, at low-frequency $(200 \mathrm{mHz})$, to produce sudden optical power changes (AM generation). Thus, we could observe the phase shift variations (PM record) induced by the photodetector and the reception electronic stage. The optical power received by the photodetector can be written as followed: 
MacroScale

Recent developments in traceable dimensional measurements Measurement Science and Technology

$$
P_{\text {opt.total }}=\underbrace{\alpha_{A O M}(t)}_{\text {squarewave mod ulated }} \times G_{E D F A} \times\left(P_{o p t . D C}+P_{o p t . R F} \cdot \cos \left(2 \pi f_{m} t+\phi_{0}\right)\right)
$$

where $\alpha_{A O M}$ is the optical attenuation induced by the AOM (a function of the power of the 80 $\mathrm{MHz}$ carrier driving it), $G_{E D F A}$ is the EDFA gain, $P_{\text {opt. }} D C$ is the average optical power at the EDFA input, $P_{\text {opt. } R F}$ is the RF optical power at the EDFA input, and $\phi_{0}$ is the phase at $\mathrm{t}=0$.

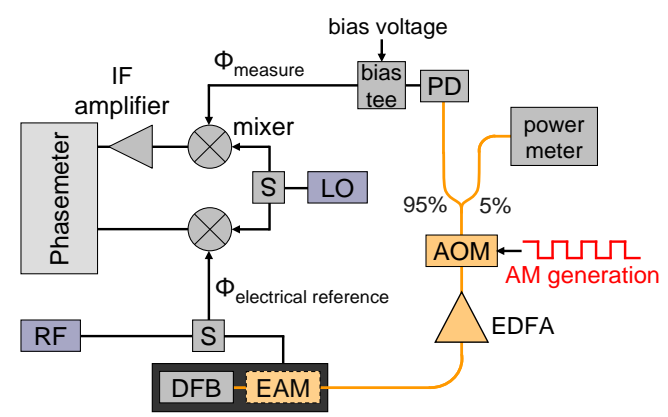

Figure 2: Setup used to characterize the AM/PM coupling occurring in the reception system.

The phase shift variations were recorded as a function of the bias voltage for different optical powers received by the photodetector (by adjusting the EDFA gain). For each record, the same square wave was applied to the AOM: the optical intensity alternated between a maximum and a minimum ( $24 \%$ lower), resulting in a power ratio of $\Delta P=1.2 \mathrm{~dB}$. At the photodiode output, the measured RF hops were, as expected, twice bigger due to square law detection.

The results have been normalized by the power ratio $\Delta P$ [9] and plotted in Fig. 3 . Additionally, we have converted for convenience the phase shift variations $\Delta \phi_{A M / P M}$ into equivalent distance variations $\Delta d_{A M / P M}$ by the following relation:

$$
\Delta d_{A M / P M}=\Delta \varphi_{A M / P M} \times \frac{c}{2 \pi \cdot f_{R F}}
$$

Fig. 3 presents the results for the MSM (a) and avalanche (b) photodiodes. The maximum optical power incident onto the avalanche photodiode is lower than the maximum power received by the MSM photodiode $(60 \mu \mathrm{W}$ versus $250 \mu \mathrm{W})$ to take into account its higher efficiency and avoiding in this way the saturation of the electronic stage.
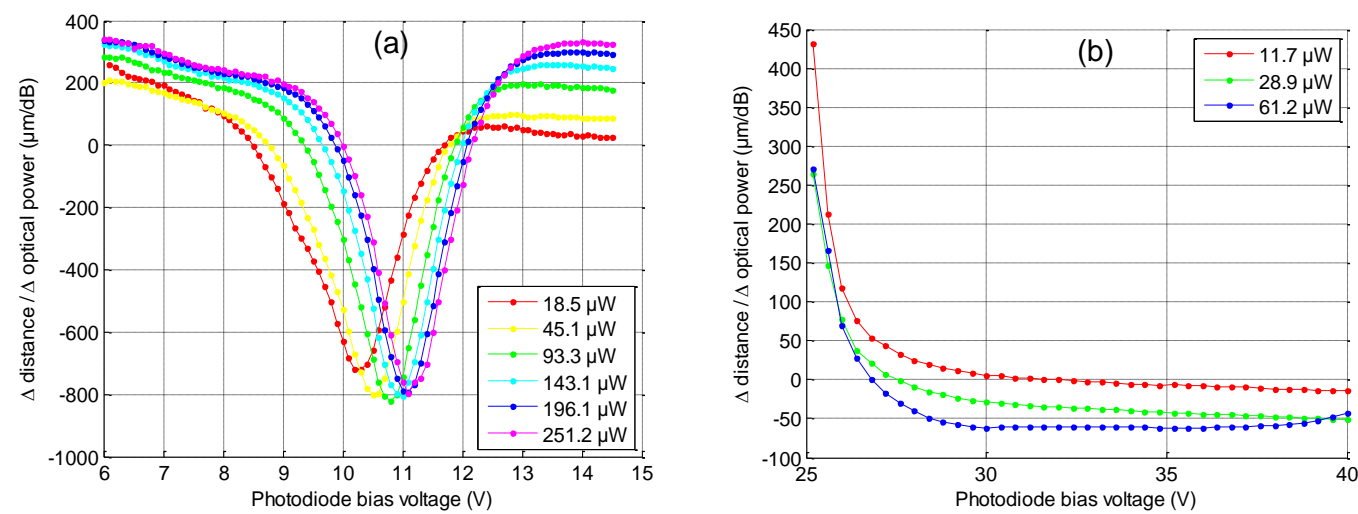

Figure 3: Phase shift variations, converted into distances, as a function of the photodiode bias voltage and for different optical powers incident onto the photodiode. The optical power values indicated in the legends correspond to the maximum values of the square optical power function. (a) MSM photodiode and (b) avalanche photodiode. 
Measurements performed on the MSM photodetector at several days intervals show a good reproducibility of the curve shape, showing two zeros. However, the bias voltages corresponding to these zeros were different from one measurement to another; this is probably due to temperature evolution in the optoelectronic components. Nonetheless for any optical power we always obtained the same curve progression with two null AM/PM coupling values. The avalanche photodiode have shown a different behaviour: the AM/PM coupling curve crosses only once the zero $y$-axis, but beyond $30 \mathrm{~V}$, it remains relatively flat. The results for the Schottky photodiode are presented in Fig. 4 (a). As the bias voltage applied to this photodiode cannot be varied due to its packaging (value fixed by the manufacturer at $9 \mathrm{~V}$ ), the distance variations have been measured only as a function of the received optical power. The results showed that the AM/PM conversion can be minimized by adjusting the incident optical power at around $100 \mu \mathrm{W}$.
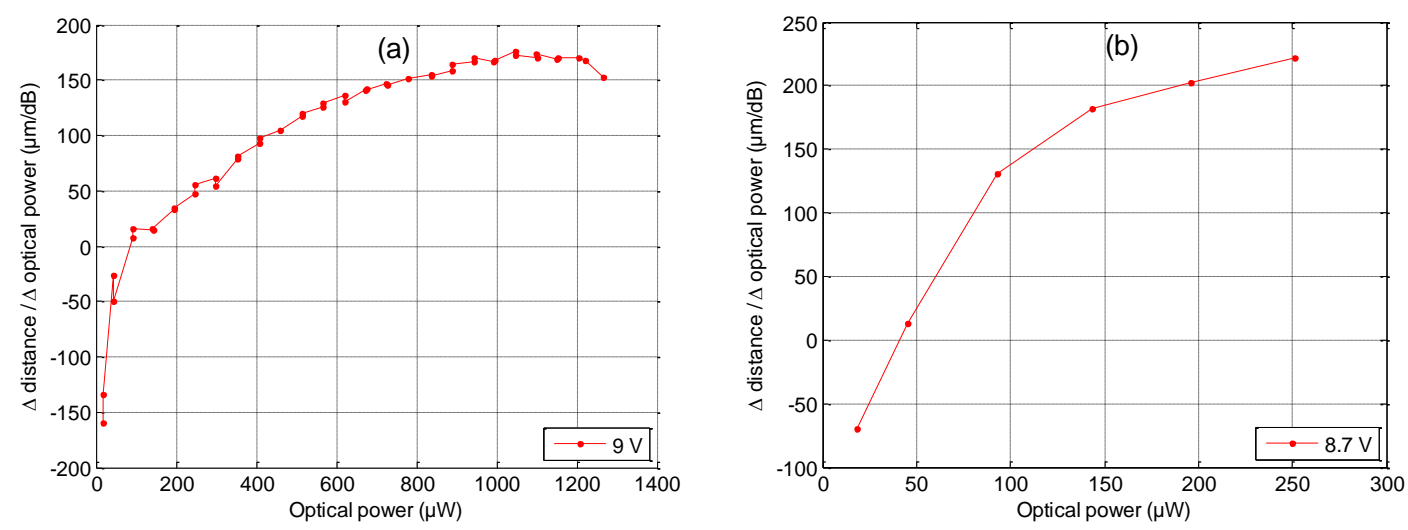

Figure 4: Distance variations as a function of the optical power incident on the photodiode for a fixed photodiode bias voltage. The optical value indicated in the $x$-axis corresponds to the maximum value of the square optical power function. (a) Schottky photodiode and (b) MSM photodiode.

As shown in Fig. 4 (b), a similar curve is obtained with the MSM photodiode for a bias voltage of $8.7 \mathrm{~V}$. This result is as expected since a MSM structure consists in two metalsemiconductor contacts (Schottky junction) connected back-to-back.

2. Distinction between the AM/PM coupling due to $P_{\text {opt. } D C}$ and the one due to $P_{\text {opt. }} R$.

For a better understanding of the phenomenon that occurs in the reception chain, we have separated the effect of the average optical power $\left(P_{\text {opt. }} D C\right.$ in Eq. 3) from the effect of the RF optical signal $\left(P_{\text {opt. } R F}\right)$. To this end, one of these parameters (either $P_{\text {opt. } D C}$ or $P_{\text {opt. } R F}$ ) was square-wave modulated (AM generation) while the second one was maintained constant (no AM). This has been realized introducing a second laser in the initial setup of Fig. 2.

\subsection{Variation of the average optical power}

A sudden optical power change was applied only on the average optical power and not on the RF modulation of the light (refer to Eq. 2). For this purpose, as shown in Fig. 5, the light from the first laser was RF modulated while the light from the second laser was low-frequency square-wave modulated. Thanks to a 50/50 optical coupler, the photodiode received the sum of the two signals as described in Eq. 5 (for clarity coupler insertion losses have been ignored). 


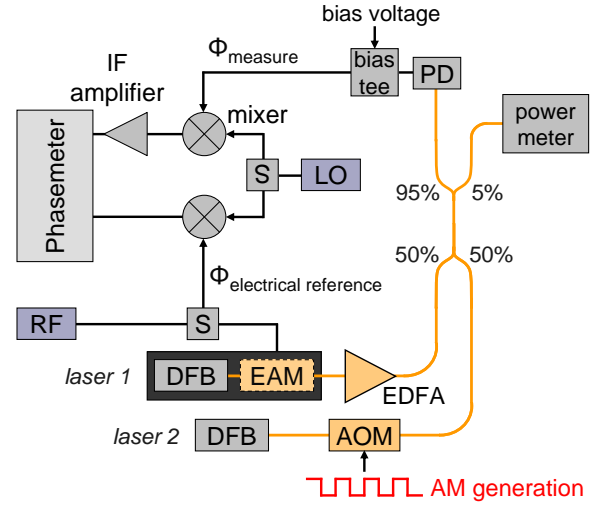

Figure 5: Setup characterizing the AM/PM coupling due to the DC optical power variations only.

$$
P_{\text {opt.total }}=\underbrace{\alpha_{\text {AOM }}(t)}_{\text {squarewave modulated }} P_{\text {optlaser } 2 D C}+G_{E D F A} \times\left(P_{\text {optlaserlDC }}+P_{\text {optlaser } 1 R F} \cdot \cos \left(2 \pi f_{m} t+\phi_{0}\right)\right)
$$

The results are presented in Fig. 6 for the MSM photodiode (a) and the avalanche photodiode (b). The shapes of these curves are similar to the previous ones in Fig. 3. Therefore, it can be concluded that the dependence of the AM/PM coupling on the bias voltage essentially comes from the photodiode, and not from the electronic stages (even if varying the photodiode bias voltage modifies the photodiode efficiency and so changes the received RF power).
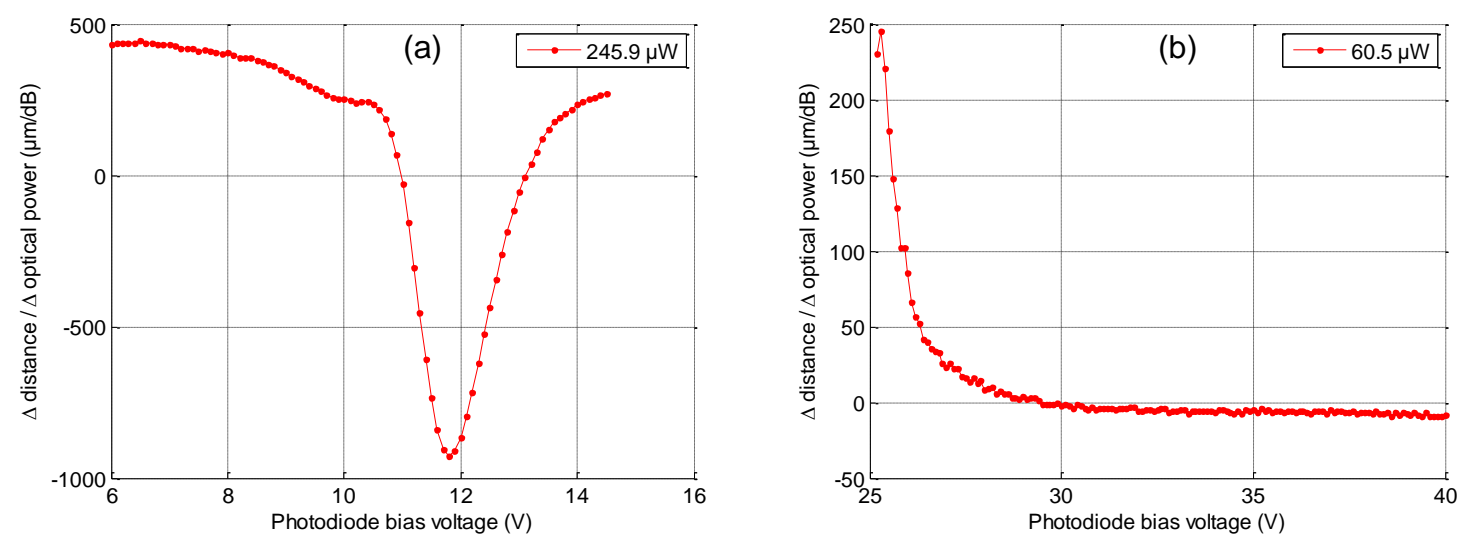

Figure 6: Distance variations as a function of the photodiode bias voltage for a constant RF optical power and a square-wave modulated DC optical power. (a) MSM photodiode in (a) and (b) avalanche photodiode.

\subsection{Variation of the RF optical power}

The reception system was then studied when only the RF modulation of the light varies. To achieve this, the setup of Fig. 7 has been realized. The arm of the first laser corresponds to the configuration depicted in Fig. 2. Thus, the average and RF optical signals are both modulated (Eq. 3). To maintain a constant average optical signal, a variable optical attenuator (ATT in Fig. 7) was placed on the second arm to counteract the square-wave modulation of the first arm. Thus, the RF power received by the photodiode and the electronic stages varies while the average optical power received by the photodiode is maintained constant. 


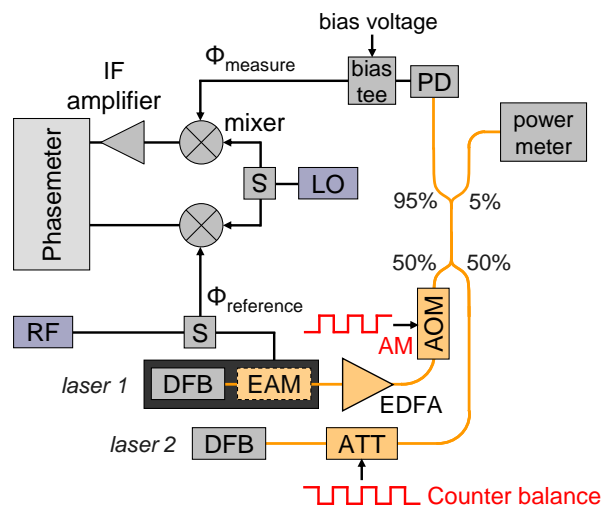

Figure 7: Setup characterizing the AM/PM coupling due to the RF optical power variations only.

The results are presented in Fig. 8. The sudden optical power changes affect only the RF modulation of the light. As a consequence, the results have been normalized by twice the electrical power ratio, which is equivalent to the previous approach.
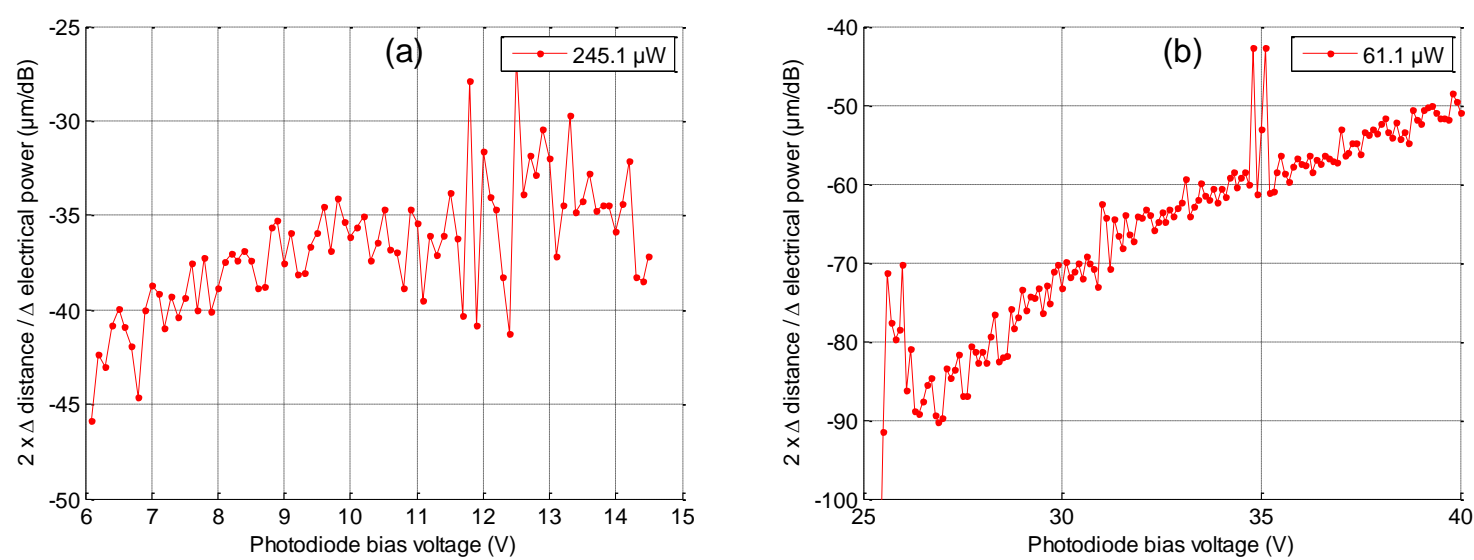

Figure 8: Distance variations as a function of the photodiode bias voltage and for a constant average optical power and a square-wave modulated RF optical power. (a) MSM photodiode and (b) avalanche photodiode.

It appears that the AM/PM coupling depends only weakly on the photodiode bias voltage. As shown in Fig. 8 (a), for the MSM photodiode, a small deviation of only $10 \mu \mathrm{m}$ is observed. This is probably induced by the electronic stages as the RF power at the photodiode output varies by $3 \mathrm{~dB}$ between 6 and $14.5 \mathrm{~V}$ due to a change of the photodiode efficiency with the bias voltage. This effect can also be observed in the case of the avalanche photodiode (Fig. 8 (b)) and is more important because the change of the photodiode efficiency is larger: $10 \mathrm{~dB}$ from 27 to $40 \mathrm{~V}$.

\subsection{Discussion}

The AM/PM conversion is not attributed only to the photodiodes, but also to the electronic stage (down-conversion and RF amplification). By adding the contribution of the photodiode (RF power constant and variations of the average optical power) to the contribution of the electronic stage only, we obtain exactly the global contribution of the reception stage to AM/PM coupling (see Fig. 9). 

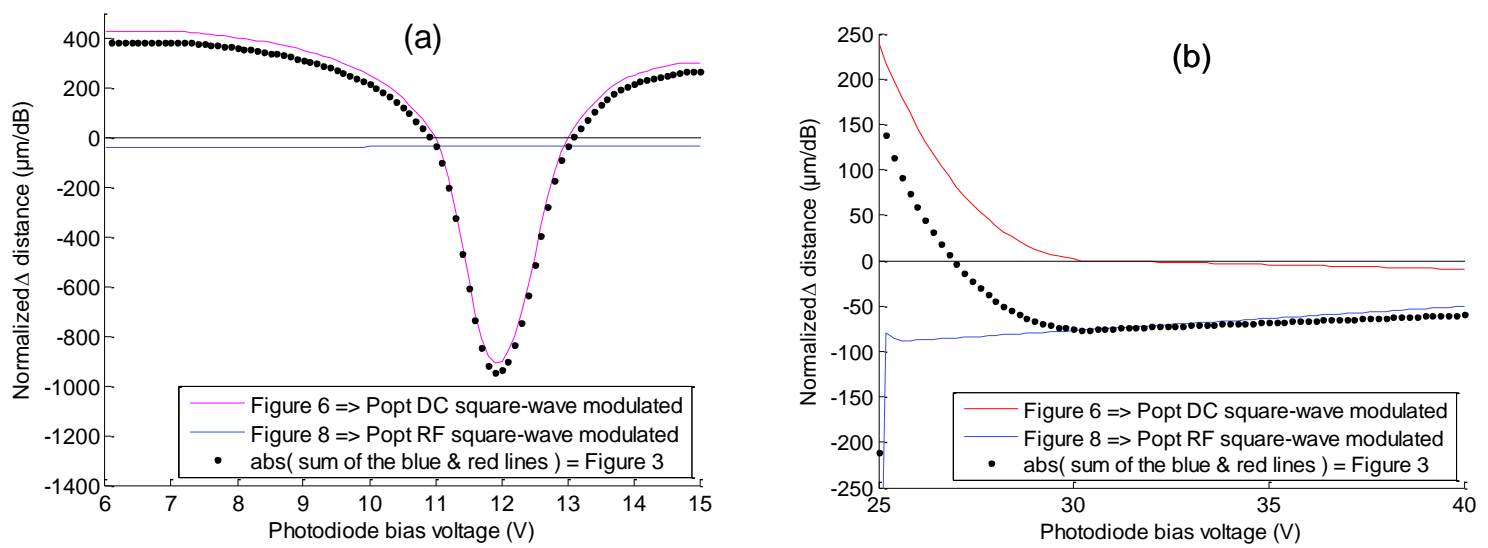

Figure 9: Summary of AM/PM conversions into the photodetectors (red lines), into the electronic stages (blue lines) and into the whole system (black dots). (a) MSM photodiode and (b) avalanche photodiode.

The MSM photodiode exhibits two bias voltages for which the AM/PM coupling is null. However, these values depend on the received optical power, and worse, they are not stable in time. Nevertheless, for bias voltages higher than $13 \mathrm{~V}$ (high photodiode efficiency) and low optical power $(20 \mu \mathrm{W})$, the whole AM/PM conversion becomes acceptable.

The avalanche photodiode shows a weaker dependence of the AM/PM coupling with the bias voltage. Indeed, for bias voltages higher than $30 \mathrm{~V}$ the avalanche photodiode itself has an acceptable AM/PM conversion (below $10 \mu \mathrm{m} / \mathrm{dB}$ for $1.3 \mathrm{GHz}$ of modulation frequency). In that case, the major part of the AM/PM conversion is induced by the electronic stages (the RF mixer and/or the amplifiers).

In both MSM and avalanche photodiodes, we did not find a stable configuration where the AM/PM can be cancelled out. Nevertheless, an appropriate data processing enables to limit the impact of this phenomenon on the resolution of the developed telemeter.

\section{Overcome the AM/PM coupling issue in telemetry application}

The digital electronic designed for phase measurements provides the amplitude of input signals additionally to the phase information. Therefore, we can select the phase shift values corresponding to a given amplitude. This approach has been tested for the MSM photodiode. In practice - as our measurement consists in a phase difference between a reference path and the measured path (Fig. 1) - we adjust the amplitude of the reference path signal (with a variable optical attenuator) to the mean value of the measurement path signal. This ensures that measurement and reference signals have the same AM/PM conversion, and so that the system provides a good accuracy.

This approach has been tested over $110 \mathrm{~m}$ outdoor along an asphalt road, during a warm (temperature around $35^{\circ} \mathrm{C}$ ) and sunny day, which implies strong air turbulences. As shown in Fig. 10 (in blue), large power fluctuations were observed with some signal cancellations $(75 \%$ of the measured power was contained within $15 \mathrm{~dB}$ ). Nevertheless the signal amplitude remained stable over a couple of ms. In these conditions, the standard deviation on the measured distances (i.e. the resolution) was $1.5 \mathrm{~mm}$. However, after filtering the data - only phase values corresponding to amplitudes equal to $7.9 \mathrm{~dB} \pm 2 \mathrm{~dB}$ (in red) was retained - the standard deviation over the measured distances was improved to $38 \mu \mathrm{m}$. This corresponds to $27 \%$ of the recorded data. By comparison, in ideal conditions, i.e. indoor measurement over $1 \mathrm{~m}$, power fluctuations are within $0.1 \mathrm{~dB}$ and a standard deviation less than $5 \mu \mathrm{m}$ was obtained. 

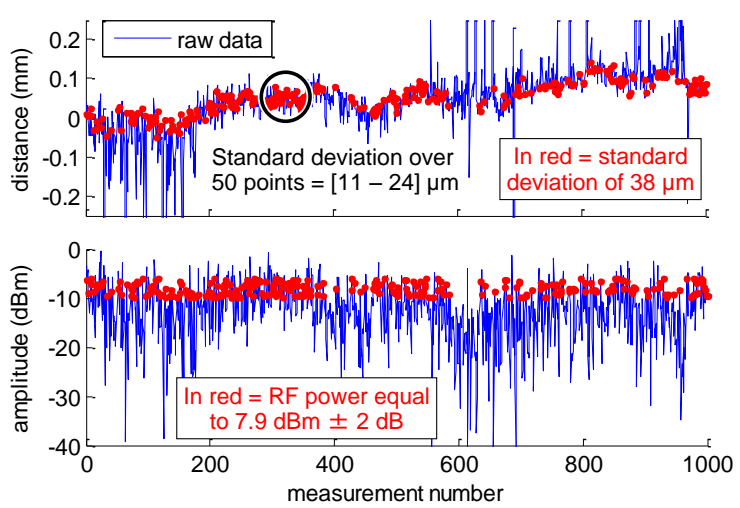

Figure 10: Distance variations and corresponding signal amplitudes of the received signal for 1000 successive distance measurements, which correspond approximately to 1000s. In blue raw data and in red data corresponding to a given $R F$ power $\pm 2 \mathrm{~dB}$.

In Fig. 10, a drift of $150 \mu \mathrm{m}$ on the measured distance can be observed. Even if we did not carry out air temperature measurement all along the optical path, this drift can be explained by an average temperature change of $1{ }^{\circ} \mathrm{C}$. Indeed, such a change should modify the air refractive index, and consequently the measured distance, by a factor $10^{-6}$ [12].

It is therefore more relevant to consider the standard deviation at short term $(50 \mathrm{~s})$ to ignore the long term drift: thus, we obtain a standard deviation comprised between 11 and $24 \mu \mathrm{m}$.

\section{Conclusion}

A small intensity noise on a RF modulated optical signal can induce an important phase noise on the RF modulation due to AM/PM conversions that occur in the photodiodes and the electronic stages. The atmospheric perturbations - which involve amplitude changes in the detected intensity-modulated beam - induce unacceptable distance variations in an accumulated-phase-shift based telemeter. We have shown for three photodetector types (MSM, avalanche and Schottky photodiodes) that two parameters are of great importance on the AM/PM conversions: the optical power received by the photodiode and the bias voltage applied to it. Thus, it is possible to find an optical power / bias voltage pair that cancels out the AM/PM coupling. Nevertheless, the photodiode bias voltage corresponding to this cancellation was not enough stable in time. So, in order to minimize this effect raw data were instead filtered to keep only the phase measurements corresponding to the same signal amplitude (the amplitude of the measurement path) within a given interval. This is possible because our digital electronic is able to capture the signals and to process them fastly (within $4 \mathrm{~ms}$ ), at a time scale where the signal level remains stable. Using this technique, we measured outdoor, with strong turbulences, a distance of $110 \mathrm{~m}$ with a short term $(50 \mathrm{~s})$ standard deviation better than $24 \mu \mathrm{m}$.

These very encouraging results are a first step towards a two-wavelength approach. The simultaneous use of visible and infrared lights in the telemeter will compensate the fluctuations of the air refractive index with temperature taking advantage of dispersion of air index [13].

\section{Reference}

[1] Rüeger J M 1990 Electronic Distance Measurement 3rd edn (Berlin: Springer)

[2] Froome K D and Bradsell R H 1966 A new method for the measurement of distances up to $5000 \mathrm{ft}$ by means of a modulated light beam J. Sci. Instrum. 43129 
MacroScale

Recent developments in traceable dimensional measurements Measurement Science and Technology

[3] Leica Absolute Tracker AT401, ASME B89.4.19-2006 Specifications. Website: www. geotech.sk/downloads/Totalne-stanice/AT401/AT401_ASME_specifikacie_presnosti_en.pdf. Accessed December 1, 2014

[4] Fujima I, Iwasaki S and Seta K 1998 High-resolution distance meter using optical intensity modulation at $28 \mathrm{GHz}$ Meas. Sci. Technol. 91049

[5] Yokoyama S, Ohnishi J, Iwasaki S, Seta K, Matsumoto H and Suzuki N 1999 Real-time and high-resolution absolute-distance measurement using a two-wavelength superheterodyne interferometer Meas. Sci. Technol. 10 1233-1239

[6] Azouigui S, Badr T, Wallerand J-P, Himbert M, Salgado J and Juncar P 2010 Transportable distance measurement system based on superheterodyne interferometry using two phase-locked frequency-doubled Nd:YAG lasers Rev. Sci. Instrum. 81053112

[7] Salvadé Y, Schuhler N, Lévêque S and Le Floch S 2008 High-accuracy absolute distance measurement using frequency comb referenced multiwavelength source Appl. Opt. 47 27152720

[8] Lay O P, Dubovitski S, Peters R D and Burger J P 2003 MSTAR: a submicrometer absolute metrology system Opt. Lett. 28 890-892

[9] Taylor J, Datta S, Hati A, Nelson C, Quinlan F, Joshi A and Diddams S, 2011 Characterization of power-to-phase conversion in high-speed P-I-N photodiodes IEEE Photon. J. 3 140-151

[10] Zhang W, Li T, Lours M, Seidelin S, Santarelli G and Le Coq Y 2012 Amplitude to phase conversion of InGaAs pin photo-diodes for femtosecond lasers microwave signal generation Appl. Phys. B 6 301-308

[11] Phung D H, Merzougui M, Alexandre C and Lintz M 2014 Phase measurement of a microwave optical modulation: characterization and reduction of amplitude-to-phase conversion in $1.5 \mu \mathrm{m}$ high bandwidth photodiodes J. Lightwave Technol. 323759 - 3767

[12] Ciddor P E 1996 Refractive index of air: New equations for the visible and near infrared Appl. Opt. 35 1566-1573

[13] Earnshaw K B and Hernandez E N 1972 Two-laser optical distance-measuring instrument that corrects for the atmospheric index of refraction Appl. Opt. 11 749-754

\section{Acknowledgements}

This project is performed within the joint research project SIB60 "Surveying" (http://www.ptb.de/emrp/sib60-home.html) of the European Metrology Research Programme (EMRP). The EMRP is jointly funded by the EMRP participating countries within EURAMET and the European Union. 